\title{
Instrumente der Vorausplanung in der Psychiatrie: Konzepte und Anwendungsfelder
}

\author{
Jakov Gather • Annemarie Heberlein · Anna-Karina Jakovljević
}

Online publiziert: 8. August 2016

(C) Springer-Verlag Berlin Heidelberg 2016

Psychische Erkrankungen gehören zu den häufigsten Krankheiten überhaupt und werden in Zukunft an gesellschaftlicher Bedeutung weiter zunehmen (8, S. 1-11). Viele dieser Erkrankungen sind durch einen episodenhaften Verlauf gekennzeichnet, bei dem sich in medizinischen Entscheidungssituationen Phasen von Einwilligungsfähigkeit mit Phasen von krankheitsbedingter Einwilligungsunfähigkeit abwechseln können. Beispielhaft seien schwere manische oder depressive Episoden im Rahmen einer bipolaren Störung, akute psychotische Zustände bei Erkrankungen aus dem schizophrenen Formenkreis oder wiederholte schwere Intoxikationen bei Suchtmittelabhängigkeit genannt. Hinzu kommen Demenzerkrankungen, die durch einen fortschreitenden kognitiven Abbau zu einem zunehmenden und schließlich dauerhaften Verlust der Fähigkeit zu selbstbestimmten Entscheidungen im medizinischen Kontext führen.

Dr. med. J. Gather, M.A. ( $\varangle)$

Klinik für Psychiatrie, Psychotherapie und Präventivmedizin, LWL-Universitätsklinikum, Ruhr-Universität-Bochum, Alexandrinenstr. 1, 44791 Bochum, Deutschland

E-Mail: jakov.gather@rub.de

Dr. med. J. Gather, M.A.

Institut für Medizinische Ethik und Geschichte der Medizin, Ruhr-Universität Bochum, Malakowturm - Markstr. 258a, 44799 Bochum, Deutschland

PD Dr. med. A. Heberlein, M.A.

Fachklinik Uchtspringe, Klinik für Gerontopsychiatrie, Emil-Kraepelin-Str. 6, 39576 Stendal, Deutschland

A.-K. Jakovljević, M.A.

Institut für Ethik und Geschichte der Medizin, Universitätsmedizin Göttingen,

Humboldtallee 36, 37073 Göttingen, Deutschland

A.-K. Jakovljević, M.A.

MRVZN Moringen, Fachkrankenhaus für Forensische Psychiatrie und Psychotherapie,

Mannenstr. 29, 37186 Moringen, Deutschland 
Um die Selbstbestimmung von Menschen mit psychischen Erkrankungen auch in Phasen von krankheitsbedingter Einwilligungsunfähigkeit zu erhalten, sprechen unter ethischen Gesichtspunkten gute Gründe dafür, Instrumente der Vorausplanung in der Psychiatrie einzusetzen [3]. Neben den seit 2009 in Deutschland gesetzlich verankerten Patientenverfügungen ( $§$ 1901a Bürgerliches Gesetzbuch) steht dazu eine Vielzahl an mehr oder weniger unterschiedlichen Instrumenten und Ansätzen zur Verfügung, die u. a. von sog. Krisenpässen über Behandlungsvereinbarungen bis hin zum Advance Care Planning reichen [1, 4, 6].

Trotz vielerorts vorhandener Initiativen von psychiatrischen Kliniken, Krankenhausträgern, gemeindepsychiatrischen Verbünden, Interessen- und Betroffenenverbänden u. v. m. zur Entwicklung und vermehrten Implementierung von Instrumenten der Vorausplanung ist mit Blick auf die klinische Realität in Deutschland jedoch nüchtern zu konstatieren, dass Vorausverfügungen auffallend selten Anwendung finden $[2,7]$. Wenngleich die Gründe hierfür vielschichtig sein mögen, scheinen viele Unsicherheiten, Missverständnisse und Vorbehalte von Professionellen wie auch von Betroffenen bereits auf einer konzeptionell-theoretischen Ebene zu bestehen. Klinische Situationen, in denen Vorausverfügungen eher als zusätzliche Herausforderung denn als hilfreiches Instrument zur erleichterten Entscheidungsfindung wahrgenommen werden (z. B. nicht selten im Zusammenhang mit zwangsweise erfolgten Unterbringungen), befördern skeptische Grundhaltungen und führen schließlich zu Zurückhaltung bei der Implementierung.

Das vorliegende Themenheft verfolgt vor diesem Hintergrund das Ziel, Konzepte und Anwendungsfelder von Instrumenten der Vorausplanung aus einer interdisziplinären Perspektive zu untersuchen und durch konzeptionell-theoretische Beiträge einerseits und die ethische Diskussion von Fällen aus der klinischen Praxis andererseits zu einem sachkundigen Umgang mit Vorausverfügungen in der Psychiatrie beizutragen. Durch die Identifizierung und Erörterung spezifischer theoretischer und praktischer Herausforderungen in der Psychiatrie soll zudem ein Beitrag zum Abbau von Hindernissen bei der Implementierung von Vorausplanungsinstrumenten geleistet werden.

\section{Die Beiträge im Einzelnen}

Das Themenheft umfasst verschiedene Beitragsformate, geschrieben von Autorinnen und Autoren mit unterschiedlichen, für die Thematik einschlägigen disziplinären Hintergründen (insbesondere Recht, Ethik und Medizin). Die meisten Autorinnen und Autoren sind Mitglieder der Arbeitsgruppe „Ethik in der Psychiatrie“ in der Akademie für Ethik in der Medizin (AEM).

Im ersten Beitrag loten Bettina Wenzel und Frauke Klinge aus einer Nutzerperspektive Chancen und Grenzen von Vorausplanungsinstrumenten in der Psychiatrie aus. In ihrem Kommentar plädieren die Autorinnen für eine Kooperation ,,auf Augenhöhe“ zwischen Nutzern und Professionellen und weisen auf vielfältige positive Effekte hin, welche die in einem offenen kommunikativen Prozess entstandenen Behandlungsvereinbarungen und Patientenverfügungen mit sich bringen können. 
In einem Übersichtsartikel von Katrin Radenbach und Alfred Simon werden unterschiedliche Formen der gesundheitlichen Vorausplanung (Advance Care Planning) in der Psychiatrie thematisiert. Dabei werden Erfahrungen mit diesen Instrumenten in den USA, Großbritannien, Deutschland und Österreich sowie die internationale Studienlage zur Anwendungspraxis in der Psychiatrie dargestellt. Diese zeigen, dass der Bedarf an qualifizierten Beratungs- und Unterstützungsangeboten in der klinischen Praxis groß ist und dass psychiatrische Vorausverfügungen bislang nur selten zur Anwendung kommen.

Im Bereich der Originalarbeiten erörtert Gunnar Duttge die Grenzen des Selbstbestimmungsrechts in der psychiatrischen Behandlungspraxis. Die Betonung des Selbstbestimmungsrechts für psychiatrische Patienten im Fall ihrer Einwilligungsunfähigkeit erfordere aus einer juristischen Perspektive eine Neujustierung der Grenzlinien zwischen Patientenwillen und der Anwendung von fürsorglichem Zwang. Die Annahme, dass im Kontext der veränderten Rechtsprechung eine Zwangsbehandlung per se unzulässig sei, beruhe auf einem reduktionistischen Autonomieverständnis. Vor diesem Hintergrund diskutiert der Autor das Instrument der psychiatrischen Behandlungsvereinbarung sowie die erforderlichen rechtlichen und institutionellen Rahmenbedingungen für die Umsetzung in der klinischen Praxis.

Jakov Gather, Tanja Henking, Georg Juckel und Jochen Vollmann diskutieren in ihrem Beitrag ethische und rechtliche Herausforderungen in klinischen Situationen, in denen psychiatrische Therapiemaßnahmen in einer Patientenverfügung zwar (gänzlich) abgelehnt werden, ein aktuell einwilligungsunfähiger Patient jedoch wegen Eigen- bzw. Fremdgefährdung gesetzlich in einem psychiatrischen Krankenhaus untergebracht ist und (vorerst) nicht entlassen werden kann. Mit Blick auf ethisch begründete Entscheidungen in der klinischen Praxis analysieren sie verschiedene Handlungsoptionen aus einer ethischen und rechtlichen Perspektive.

Anna-Karina Jakovljevic, Dirk Hesse und Claudia Wiesemann thematisieren in ihrem Beitrag die Anwendungspraxis von Patientenverfügungen und Behandlungsvereinbarungen in der forensischen Psychiatrie und diskutieren, ob und wie diese Instrumente in der forensischen Behandlung eingesetzt werden können und welche zusätzlichen Maßnahmen zur Stärkung der Patientenselbstbestimmung erforderlich sind. Anhand eines Fallbeispiels werden Folgen einer Behandlungsablehnung für den Patienten und das Behandlungsteam skizziert und es wird aus einer medizinethischen Perspektive analysiert, welche spezifischen Anforderungen die forensische Psychiatrie an Instrumente der Vorausplanung im klinischen Alltag stellt.

Manuel Trachsel und Nikola Biller-Andorno fokussieren in ihrem Beitrag auf die Anwendung von Patientenverfügungen bei einer unfreiwilligen Unterbringung in einer psychiatrischen Klinik in der Schweiz. Patientenverfügungen für psychiatrische Behandlungssituationen müssen in der Schweiz im Behandlungsplan lediglich berücksichtigt werden und sind nicht - wie entsprechende Vorausverfügungen für somatische Behandlungssituationen - rechtlich verbindlich. Die Autoren analysieren aus einer medizinethischen Perspektive mögliche Gründe für diese Ungleichbehandlung, die die Selbstbestimmung von psychiatrischen Patienten in Frage stelle und eine Stigmatisierung dieser besonders vulnerablen Patientengruppe zur Folge haben könne. 
In der Rubrik „Fall und Kommentare“ wird die Umsetzung einer Behandlungsvereinbarung in der stationären psychiatrischen Behandlung am Beispiel eines Patienten mit einer akuten Psychose diskutiert. In Kommentar I verweisen Tanja Müller und Susanne Markwort auf die Herausforderung, bei der Erstellung einer Behandlungsvereinbarung mit einem Patienten Kompromisse einzugehen, die dann nachher von betroffenen Behandlungsteams mitgetragen werden müssen. Sie plädieren für eine frühzeitige Erörterung der Konsequenzen einer Behandlungsvereinbarung mit dem jeweiligen Patienten. In Kommentar II analysiert Orsolya Friedrich ethische Aspekte der klinischen Umsetzung der Behandlungsvereinbarung und hinterfragt anhand des Fallbeispiels kritisch ihre Zielsetzung und ihren Mehrwehrt im Vergleich zu einer Patientenverfügung.

In einer Kontroverse diskutieren schließlich Sabine Müller (Pro) und Elsa Romfeld (Contra) die Anwendung von Odysseus-Verfügungen - einer besonderen Variante der Vorausverfügung, in der der Verfasser einen späteren, seiner Verfügung entgegenstehenden ,natürlichen“ Willen für unbeachtlich erklären möchte [5] - unter besonderer Berücksichtigung der tiefen Hirnstimulation (THS). Sabine Müller argumentiert für die Anwendung von Odysseus-Verfügungen und stützt ihre Argumentation unter anderem auf den Schutz des autonomen Willens und die Möglichkeit, trotz im Verlauf entstandener krankheitsbedingter Willensveränderungen eine erforderliche und gewünschte medizinische Diagnostik und Therapie erhalten zu können. Elsa Romfeld hingegen hinterfragt kritisch die in Odysseus-Verfügungen zum Ausdruck gebrachte grundsätzliche Höherbewertung eines früheren selbstbestimmten im Vergleich zu einem späteren „,natürlichen“ Willen. Im Zusammenhang mit einer THS-Behandlung diskutiert sie zudem das spezifische Problem, dass durch THS-verursachte Persönlichkeitsveränderungen zwei „kompetente“ Willensäußerungen (nämlich bei „THS ein“ bzw. bei „THS aus“) miteinander in Konflikt geraten können.

\section{Danksagung}

Unser Dank gilt zunächst den Autorinnen und Autoren der einzelnen Beiträge für die gute und kollegiale Zusammenarbeit. Den Kolleginnen und Kollegen der AG „Ethik in der Psychiatrie“ sei für die Diskussion der in diesem Heft versammelten Themen bei vergangenen Arbeitstreffen gedankt. Den externen Gutachterinnen und Gutachtern danken wir für ihre kritisch-konstruktiven Begutachtungen. Der Schriftleitung von Ethik in der Medizin - namentlich Thomas Schramme und Ralf Jox möchten wir für ihre Arbeit und Unterstützung danken. Unser besonderer Dank gebührt Andrea Kähling, die durch ihre kontinuierliche und sorgfältige redaktionelle Arbeit und Beratung einen wichtigen Beitrag zur Realisierung dieses Themenhefts geleistet hat. 


\section{Literatur}

1. Birnbacher D (2016) Patientenverfügungen und Advance Care Planning bei Demenz und anderen kognitiven Beeinträchtigungen. Ethik Med doi:10.1007/s00481-016-0388-6

2. Borbé R, Jaeger S, Borbé S, Steinert T (2012) Anwendung psychiatrischer Behandlungsvereinbarungen in Deutschland. Ergebnisse einer bundesweiten Befragung. Nervenarzt 83:638-643

3. Deutsche Gesellschaft für Psychiatrie und Psychotherapie, Psychosomatik und Nervenheilkunde e.V. (DGPPN) (2014) Achtung der Selbstbestimmung und Anwendung von Zwang bei der Behandlung psychisch erkrankter Menschen. Eine ethische Stellungnahme der DGPPN. Nervenarzt 85:1419-1431

4. Henderson C, Swanson JW, Szmukler G, Thornicroft G, Zinkler M (2008) A typology of advance statements in mental health care. Psychiatr Serv 59(1):63-71

5. Henking T, Bruns H (2014) Die Patientenverfügung in der Psychiatrie. Z Gesundheitsr 10:585-590

6. Kühlmeyer K, Borbé R (2015) Vorausplanung und Vorausverfügung in der Psychiatrie. In: Coors M, Jox RJ, in der Schmitten J (Hrsg) Advance Care Planning. Von der Patientenverfügung zur gesundheitlichen Vorausplanung. Kohlhammer, Stuttgart, S 328-341

7. Radenbach K, Falkai P, Weber-Reich T, Simon A (2014) Joint crisis plans and psychiatric advance directives in German psychiatric practice. J Med Ethics 40:343-345

8. Schneider F, Falkai P, Maier W (2012) Psychiatrie 2020 plus. Perspektiven, Chancen und Herausforderungen. Springer, Heidelberg 